\title{
ECOLOGIA FUNCIONAL COMO FERRAMENTA PARA PLANEJAR E MONITORAR A RESTAURAÇÃO ECOLÓGICA DE ECOSSISTEMAS
}

\author{
Milena Fermina Rosenfield ${ }^{1, *}$ \& Sandra Cristina Müller ${ }^{2}$
}

\author{
${ }^{1}$ University of Guelph, School of Environmental Sciences, 50 Stone Road East, N1G 2W1. Guelph, ON, Canada. \\ ${ }^{2}$ Universidade Federal do Rio Grande do Sul, Instituto de Biociências, Departamento de Ecologia, Avenida Bento \\ Gonçalves, 9500, CEP 91501-970. Porto Alegre, RS, Brasil. \\ E-mails: milenarosenfield@gmail.com (*autor correspondente); sandra.muller@ufrgs.br
}

\begin{abstract}
Resumo: A ecologia funcional tem se mostrado uma ferramenta importante em diversos estudos ecológicos. Atributos funcionais respondem a filtros ambientais e influenciam propriedades e funções ecossistêmicas, podendo ser aplicados em estudos ecológicos voltados à restauração de ecossistemas. No presente estudo apresentamos como a ecologia funcional pode ser aplicada no âmbito da ecologia da restauração, desde o planejamento até o monitoramento dos projetos, com foco no funcionamento dos ecossistemas. $\mathrm{O}$ estudo primeiramente contextualiza a teoria ecológica associada à interligação entre as disciplinas de ecologia da restauração, funcionamento de ecossistemas e ecologia funcional. Em seguida, apresentamos os resultados de uma pesquisa bibliográfica sistemática e descrevemos como a ecologia baseada em atributos funcionais tem sido aplicada no planejamento e monitoramento de projetos de restauração. Na fase de planejamento, a ecologia funcional auxilia na escolha das espécies com base em suas características, levando em conta as condições ambientais e os potenciais filtros para o desenvolvimento das espécies, bem como as metas propostas para o projeto de restauração. Na fase de monitoramento, os atributos podem ser empregados na avaliação da funcionalidade do ecossistema, dadas as relações entre atributos e processos ecológicos. Concluímos indicando que é importante focar não só na composição florística das comunidades, mas principalmente na manutenção e restauração das funções ecossistêmicas, especialmente frente ao cenário de mudanças climáticas e de uso do solo. A interligação entre restauração ecológica e funcionamento dos ecossistemas (objetivo comum em projetos de restauração) pode ser viabilizada através de abordagens baseadas em atributos funcionais, aumentando a efetividade na aplicação de recursos e o sucesso das ações de restauração.
\end{abstract}

Palavras-chave: atributos funcionais; funcionamento de ecossistemas; ecologia da restauração; processos ecológicos.

FUNCTIONAL ECOLOGY AS A TOOL FOR PLANNING AND MONITORING ECOSYSTEMS RESTORATION: Functional ecology is an important tool in several ecological studies. Functional traits respond to environmental filters and influence ecosystem properties and functions, enabling its application in ecological studies associated with ecosystem restoration. In the present study we present how functional ecology can be applied in restoration ecology, from the planning to the monitoring phases of the project, with the focus on ecosystem functioning. First, the study contextualizes the ecological theory related to the integration of the disciplines of restoration ecology, ecosystem functioning and functional ecology. From that, we present the results of a systematic review of the literature and describe how trait-based ecology has been applied in the planning and monitoring phases of restoration projects. In the planning phase functional ecology helps 
in selecting species based on their characteristics, considering environmental conditions and potential filters to species development, as well as the targeted aims of restoration projects. In the monitoring phase functional traits can be used in the evaluation of ecosystem functionality, given the relationship between traits and ecological processes. We conclude indicating that it is important to focus not only on community floristic composition, but especially on the maintenance and restoration of ecosystem functions, particularly, in light of the current scenario of climate and land-use change. The integration between restoration ecology and ecosystem functioning - a common goal in ecological restoration projects - can be made through traitbased ecology approaches, increasing the effectiveness in the use of resources and the success of restoration interventions.

Keywords: functional traits; ecosystem functioning; restoration ecology; ecological processes.

\section{INTRODUÇÃO}

A restauração ecológica é uma ciência aplicada que integra diversas áreas da ecologia (Young 2000), com propósito de restabelecer condições abióticas e características bióticas de áreas degradadas. Os estudos de restauração ecológica inicialmente tiveram foco na estrutura e diversidade da comunidade local de plantas (Rodrigues \& Leitão Filho 2001, Kageyama et al. 2003), porém, mais recentemente, a avaliação do funcionamento do ecossistema tem sido considerada essencial (Kollmann et al. 2016, Bird \& Nimmo 2018). O funcionamento do ecossistema está relacionado aos processos e às funções ecológicas decorrentes de interações entre espécies e destas com o ambiente. Assim, espera-se que o ecossistema em restauração consiga restabelecer trajetórias sucessionais inerentes ao seu tipo (seja ele florestal, campestre, aquático, etc.), após um certo período de intervenções humanas (ações de restauração).

Embora existam muitos estudos que indicam uma associação entre funcionamento dos ecossistemas e diversidade/riqueza de espécies (Tilman et al. 1996, Cardinale et al. 2007), pesquisas recentes ressaltam que não é o número de espécies, mas sim as características dessas espécies que influenciam os processos ecológicos (Loreau et al. 2001, Hooper et al. 2005, Díaz et al. 2007). Nesse sentido, a ecologia funcional, através do uso de atributos funcionais, constitui uma ferramenta importante para identificar características que afetam o funcionamento e o desenvolvimento dos ecossistemas. Sua utilização, associada a métricas de estrutura da vegetação e composição de espécies, pode gerar bons resultados na condução das ações iniciais de restauração (de acordo com metas específicas) e na avaliação das trajetórias sucessionais e do sucesso da restauração. Neste trabalho, brevemente contextualizamos as disciplinas de restauração ecológica, funcionamento de ecossistemas e ecologia funcional para então conectar suas abordagens em termos de planejamento e monitoramento de projetos aplicados à restauração ecológica. $\mathrm{O}$ objetivo principal é apresentar como a ecologia baseada em atributos funcionais pode ser usada para auxiliar no planejamento e monitoramento de projetos de restauração no contexto da recuperação de funções ecossistêmicas.

\section{Restauração ecológica}

A restauração ecológica é definida como o processo que consiste em auxiliar a recuperação de um ecossistema que foi degradado, impactado ou destruído (Society for Ecological Restoration SER 2004). Trata-se de uma atividade intencional que inicia ou acelera a recuperação do ecossistema no que diz respeito aos processos funcionais, composição de espécies, estrutura da comunidade e resistência/resiliência a distúrbios. Assim, espera-se que o ambiente restaurado apresente ausência de ameaças, condições físicas do substrato e composição de espécies adequados, diversidade estrutural da vegetação, funcionalidade do ecossistema e conectividade na paisagem (McDonald et al. 2016). As condições almejadas devem levar em conta o ecossistema que ocorria antes do evento de degradação, ou seja, o ecossistema de referência (Higgs et al. 2014). Porém, por mais que o ecossistema de referência represente uma base de comparação, um guia para a avaliação do projeto de restauração, existe a possibilidade de múltiplas trajetórias. Isso se 
deve ao fato de que tanto a trajetória sucessional (Mesquita et al. 2015, Norden et al. 2015), quanto a dinâmica dos ecossistemas de referência ao longo do tempo (Hiers et al. 2012) podem ser pouco previsíveis.

A ecologia da restauração utiliza conceitos teóricos de outras áreas da ecologia, como sucessão ecológica, fragmentação, dinâmica de comunidades, interações bióticas, entre outros, para embasar práticas que visam reduzir os danos ambientais decorrentes da degradação e conduzir o sistema à sucessão almejada. A prática da restauração ecológica engloba diferentes etapas, desde o planejamento das ações, estratégias e recursos disponíveis, passando pela execução do projeto e monitoramento ao longo do tempo (Holl \& Aide 2011, Overbeck et al. 2016). O monitoramento é essencial para verificar se o ecossistema se recuperou e se ele é resiliente diante de distúrbios comuns aos sistemas, mantendo a sua dinâmica sem auxílio externo (novas intervenções humanas). Os parâmetros para avaliar e monitorar áreas em processo de restauração dependem do objetivo do projeto e vão desde parâmetros estruturais da comunidade (ex. área basal, abundância, indicadores de composição taxonômica e funcional) até variáveis relativas ao ecossistema (processos ecológicos, ex. interações bióticas, ciclagem de nutrientes) e a questões socioeconômicas (Ruiz-Jaen \& Aide 2005, Reid 2015, Suding et al. 2015, Suganuma \& Durigan 2015, Brancalion \& Holl 2016). Como as trajetórias sucessionais são imprevisíveis e muitos são os estados possíveis para o desenvolvimento do sistema (Norden et al. 2015), que podem ou não se aproximar da meta estabelecida com base na referência, somente com monitoramento é possível avaliar como os parâmetros mudam após as intervenções de restauração e ao longo da sucessão (Suganuma \& Durigan 2015). Além disso, o monitoramento também permite identificar a necessidade de atividades de manejo adaptativo, que visam a superação de barreiras bióticas ou abióticas e o estímulo ao desenvolvimento sucessional do ecossistema (Durigan \& Ramos 2013).

Há uma necessidade legal de restaurar áreas degradadas, que vai desde acordos internacionais para promover a restauração (Chazdon et al. 2017), como o da Convenção pela Diversidade
Biológica (através das metas de Aichi; www.cbd. int) e o Desafio de Bonn (lançado pela IUCN; www. bonnchallenge.org), acordos nacionais, como o Pacto pela Restauração da Mata Atlântica - PACTO (Calmon et al. 2011; www.pactomataatlantica. org.br), até legislações específicas nacionais e regionais. No Brasil, a Lei no 12.651/2012 (Lei de Proteção da Vegetação Nativa), complementada pelo PLANAVEG (Plano Nacional de Recuperação da Vegetação Nativa), aborda a necessidade e importância da recuperação/restauração da vegetação nativa. No âmbito estadual, o Estado de São Paulo é um bom exemplo no desenvolvimento de regulamentação para promover a restauração de áreas degradadas. Através da Resolução SMA $n^{\circ} 32 / 2014$, ele apresenta diretrizes específicas para elaboração, execução e monitoramento de projetos de restauração ecológicas em seu território (Chaves et al. 2015). É importante implementar ações de restauração para que áreas degradadas, que não proporcionam qualquer benefício direto ou indireto, possam prover serviços e manter a biodiversidade.

\section{Funcionamento dos ecossistemas}

O funcionamento dos ecossistemas está relacionado aos processos e funções ecológicas, que envolvem interações entre componentes bióticos e abióticos dos ecossistemas, bem como relações estritamente bióticas, sendo indispensáveis para a manutenção do ecossistema. Esses processos envolvem transferência de energia ou matéria, sendo estimados em termos de taxas (ex. produção primária ou decomposição) ou via avaliações quali-quantitativas de múltiplas espécies (ex. regeneração natural ou dispersão de sementes). Os processos ecológicos são a base para a geração dos serviços ecossistêmicos, que são ditos os benefícios que os seres humanos obtém dos ecossistemas (MA 2005) ou ainda as contribuições diretas e indiretas dos ecossistemas para o bem estar humano (TEEB 2010). O desenvolvimento dos processos ecológicos está associado à ocorrência de diversos grupos de organismos em um dado ambiente físico e a funcionalidade associada a esses processos está ligada à capacidade de resiliência do ecossistema frente a distúrbios e à sustentabilidade ao longo do tempo.

O funcionamento dos ecossistemas pode ser afetado pela riqueza/diversidade de espécies 
(Tilman et al. 2014), conforme demonstram diversas pesquisas que abordam esse tema (BEF, do inglês Biodiversity and Ecosystem Functioning, Tilman et al. 1997). Experimentos com manipulação da riqueza/composição de espécies herbáceas indicam um efeito positivo de comunidades mais ricas na produtividade e ciclagem de nutrientes (Tilman et al. 1996, Loreau et al. 2001, Jochum et al. 2019). Esses experimentos focam especialmente nas relações em ecossistemas campestres (Buchmann et al. 2018), em virtude da maior facilidade de manipulação e montagem das comunidades. Tais relações, entretanto, têm sido observadas em outros ecossistemas, como é o caso de florestas (ex. Ruiz-Jaen \& Potvin 2010, Fujii et al. 2017). Como potencial explicação, mecanismos relacionados à complementaridade de nicho sugerem que o aumento dos valores de biodiversidade implica em uma maior variação entre espécies, permitindo o uso mais eficiente dos recursos disponíveis e, consequentemente, maiores níveis de produtividade e ciclagem de nutrientes (Loreau 1998, Loreau \& Hector 2001). Embora diversos estudos indiquem uma relação entre o número de espécies e processos ecológicos, acredita-se que não é exatamente a quantidade de espécies a responsável por essa relação, mas sim as características que esses diferentes organismos apresentam (Loreau et al. 2001, Hooper et al. 2005, Díaz et al. 2007, Rosenfield \& Müller 2020).

\section{Ecologia funcional}

A ecologia funcional ou ecologia baseada em atributos (Shipley et al. 2016) se refere aos estudos que envolvem o uso de atributos funcionais aplicados a diferentes níveis de organização de comunidades e ecossistemas. Atributos (traits, em inglês) são características descritas por diferentes valores ou estados (attribute, em inglês), associadas ao desempenho de um organismo e sua influência no ecossistema (Garnier et al. 2016). A síntese de informações relativas a um ou vários atributos para espécies que co-ocorrem em comunidades, cujos desempenhos (ex. abundâncias) variam conforme condições ambientais e/ou mecanismos de coexistência, é um dos principais focos da ecologia baseada em atributos. A ideia é compreender e predizer como a expressão funcional (estado médio e/ou variação) se associa a diferentes estruturas da comunidade e/ou ao funcionamento dos ecossistemas. A capacidade de predizer tais relações é essencial para a aplicação da ecologia funcional na ecologia da restauração.

Atributo ou traço funcional é uma característica do organismo que afeta o fitness individual ou da espécie via efeitos no crescimento, reprodução ou sobrevivência (Violle et al. 2007), respondendo a condições ambientais ou afetando propriedades do ecossistema (Lavorel 2013). Autilização de atributos permite organizar espécies ou indivíduos em grupos com funções ou respostas similares (i.e., cujos estados dos atributos são semelhantes). Conforme os atributos variam em resposta a mudanças ambientais (filtros ambientais ou biológicos) ou afetam processos ecossistêmicos, eles podem ser denominados atributos resposta ou atributos de efeito (Lavorel \& Garnier 2002). Atributos resposta são aqueles cujos valores ou estados se alteram conforme características ambientais, como por exemplo, aumento do tamanho foliar em regiões onde há maior temperatura e disponibilidade hídrica (Wright et al. 2017). Atributos de efeito são aqueles que influenciam funções ecológicas, como por exemplo, alto conteúdo de massa seca foliar afeta (reduz) a taxa de decomposição e ciclagem de nutrientes no solo (Freschet et al. 2012). Conforme o foco de cada estudo, certos atributos podem ser utilizados tanto para explicar respostas a determinados fatores ambientais ao longo de gradientes, como para compreender os efeitos sobre processos ecossistêmicos (Lavorel \& Garnier 2002, Garnier et al. 2004). Portanto, dada a relação mais direta entre atributos funcionais e propriedades ecossistêmicas (quantidade/ estrutura e fluxo de matéria e energia), os atributos funcionais podem ser usados como proxy para inferir funções e serviços no nível do ecossistema (Loreau et al. 2001, Díaz et al. 2007).

Atributos funcionais de plantas têm sido amplamente utilizados, principalmente devido à disponibilidade de informações em bases de dados globais (ex. Chave et al. 2009, Kattge et al. 2011) e regionais (ex. Galetti et al.2011, Oliveira et al. 2019). Entre os atributos mais comumente medidos estão os foliares (ex. área foliar específica, espessura, conteúdo de massa seca, conteúdo de nitrogênio e fósforo foliar), reprodutivos (ex. tamanho e tipo de fruto, massa de semente, forma de dispersão e polinização), de crescimento (ex. densidade da madeira, altura máxima potencial) e de raiz 
(ex. densidade e comprimento de raiz) (PerezHarguindeguy et al. 2013). A escolha dos atributos é uma etapa importante que afeta os resultados das ações de restauração, uma vez que determinados atributos respondem a diferentes condições e recursos, bem como têm efeitos distintos em processos ecológicos.

As funções associadas aos atributos funcionais podem ser quantificadas em diferentes níveis de organização, desde indivíduos até comunidades e ecossistemas (Garnier et al. 2016). Desta maneira, a informação dos atributos medidos no nível do organismo deve ser escalonada para outros níveis de organização, se o interesse é inferir ou compreender funções que influenciam (ou caracterizam) comunidades e ecossistemas. O escalonamento para o nível da comunidade é feito por meio de parâmetros que refletem a composição (dominância) e a diversidade funcional. De forma mais específica, são comumente utilizados: valores médios ponderados na comunidade - CWM (do inglês community weighted mean traits; Garnier et al. 2004), em que os valores são ponderados pela abundância das espécies e refletem a dominância do atributo na comunidade; ou índices de diversidade funcional - FD (do inglês functional diversity), que medem a amplitude de variabilidade do atributo na comunidade (Ricotta \& Moretti 2011), como por exemplo, entropia de Rao, riqueza, redundância e divergência funcional (Mason et al. 2005). Esses parâmetros consideram, portanto, a informação dos atributos e a composição de espécies na comunidade, a qual pode ser descrita por presença/ausência (dados binários, qualitativos) ou abundância (dados quantitativos de densidade, cobertura, biomassa, etc.). A inclusão de dados de abundância permite que a informação associada à quantidade ou massa de indivíduos de cada espécie que compõe a comunidade seja incorporada ao parâmetro funcional. Diante disso, os índices de dominância e de diversidade funcional em geral estão associados a respostas ecológicas diferentes: valores de CWM respondem a questões relativas à teoria de razão de massa (do inglês mass-ratio theory; Grime 1998), que sugere que são os atributos das espécies dominantes que mais afetam os processos ecológicos associados aos serviços ecossistêmicos (Díaz et al. 2007); enquanto índices de diversidade funcional estão mais associados a processos de montagem de comunidades e amplitude no uso de recursos, como respostas ao efeito da complementariedade de nicho (Loreau 1998, de Bello et al. 2010).

\section{MATERIAL E MÉTODOS}

Além de uma revisão geral associada aos tópicos introduzidos acima, realizamos uma busca sistemática para identificar as publicações científicas que utilizaram atributos funcionais no contexto da restauração ecológica, servindo de base e fonte de exemplos para a discussão. A busca foi feita em agosto de 2019, na plataforma Web of Science (sem restrição quanto ao ano de início), com as seguintes palavras-chave (localizadas no título, resumo e palavras-chave): "functional AND restoration AND ecolog* AND plant" e "trait OR group". Utilizamos o termo 'group' para incluir trabalhos que usam a terminologia de grupos funcionais, em que grupos de espécies são diferenciados de modo qualitativo, como por exemplo, por grupos sucessionais, como pioneiras, secundárias e tardias. Um total de 437 artigos foram inicialmente selecionados. Após avaliação, removemos os artigos que não eram sobre restauração (129 artigos faziam apenas menção a uma possível aplicação para restauração), que não focavam em atributos/grupos funcionais de plantas (149), que eram exclusivamente de revisão (17) ou puramente teóricos (10). Adicionalmente, excluímos trabalhos de congresso/conferência (2) e aqueles não disponíveis para acesso (1). Essa filtragem resultou em 129 artigos, dos quais obtivemos informações relativas a: (1) foco do estudo - teórico/experimental, planejamento, monitoramento ou banco de dados; (2) país onde o estudo foi realizado (para identificar estudos conduzidos no Brasil); (3) tipo de ecossistema - aberto (ex. campo, savana), florestal (floresta, bosque), aquático (ex. banhado) ou semi-natural (ex. margens de rodovia, ambientes minerados); (4) tipo de descritor funcional - atributo ou grupo; (5) característica do descritor - quantitativa ou qualitativa. Para determinar quais descritores foram considerados atributos funcionais, utilizamos as categorias apresentadas em Perez-Harguindeguy et al. (2013). Além dos manuscritos resultantes da revisão sistemática, o presente trabalho se baseia em artigos clássicos e recentes, de cada grande área de pesquisa (restauração ecológica, funcionamento 
de ecossistemas e ecologia funcional), publicados em revistas científicas nacionais e internacionais.

\section{RESULTADOS E DISCUSSÃO}

\section{Revisão sistemática}

A revisão sistemática resultou na avaliação de um total de 129 artigos (Material Suplementar). Dentre os 17 estudos realizados no Brasil, 16 foram realizados em florestas (Mata Atlântica, 13; Amazônia, 2; e Cerrado, 1) e apenas 1 em ecossistema aberto (savana, no Cerrado). Nenhum estudo incluído na nossa revisão foi realizado nos biomas Caatinga, Pampa ou Pantanal. Dentre os 16 estudos realizados no Brasil com florestas, 9 utilizaram atributos e 7 utilizaram grupos funcionais para descrever/categorizar as espécies. A síntese da quantidade de estudos entre as diferentes categorias da revisão é apresentada no material suplementar.

\section{Ecologia funcional no planejamento da restauração}

Considerando o que foi apresentado, entendemos que a ecologia funcional pode ser diretamente aplicada nas ações de restauração ecológica, já desde a etapa de planejamento dos projetos. Na etapa de planejamento da restauração, tanto atributos resposta, quanto atributos de efeito das espécies que serão inseridas em uma área degradada podem ser considerados, a depender do objetivo e metas do projeto, bem como das condições e histórico de uso da área (Figura 1). Por exemplo, o uso de espécies fixadoras de nitrogênio em áreas impactadas e com longo histórico de uso do solo resulta em maiores taxas de sobrevivência e crescimento individual, permitindo o estabelecimento de uma estrutura de dossel em curto prazo e gerando condições para o desenvolvimento subsequente da regeneração natural (Holl et al. 2011). Atributos individuais ou em combinações podem ser usados para prever a resposta das espécies em termos de estabelecimento inicial e recobrimento (Martínez-Garza et al. 2013), aumentando a eficiência das ações de restauração. Por outro lado, atributos de efeito podem ser priorizados dependendo do objetivo principal do projeto de restauração. Através de fundamentações claras da relação processos ecológicos $v s$. atributos funcionais já existentes na literatura, é possível selecionar espécies para plantio em áreas de restauração baseado em características-alvo do projeto (Laughlin 2014, Ostertag et al. 2015, Rosenfield \& Müller 2017). Por exemplo, se o interesse é acelerar a taxa de decomposição em um determinado ecossistema, então uma das ações de manejo seria focar em compor uma comunidade cujas espécies apresentem maiores valores de SLA e menores valores de LDMC, associados a maiores taxas de decomposição (Laughlin et al. 2010). $\mathrm{Ou}$ ainda, como exemplificado acima, o mesmo atributo resposta (espécie fixadora de nitrogênio) pode gerar efeitos em nível ecossistêmico, afetando a produção de biomassa e a ciclagem de nutrientes. Esse tipo de ação pode ser implementado desde as primeiras intervenções de restauração ou sob a perspectiva do manejo adaptativo, sendo adotadas na medida em que resultados de monitoramento indicam desvios na trajetória de desenvolvimento almejado.

A revisão sistemática indicou diversos estudos que utilizaram atributos funcionais no planejamento de projetos de restauração, na expectativa de aumentar o sucesso das ações de restauração ou combater ameaças, tais como invasão de espécies exóticas (Hallett et al. 2017) e erosão (Bochet \& Garcia-Fayos 2015, Zhu etal. 2015), especialmente considerando atributos associados ao estabelecimento e à coexistência das espécies. Atributos foliares (LA, SLA, LDMC), de crescimento (altura) e reprodutivos (massa de semente, tamanho da flor, tamanho do fruto) têm sido utilizados para determinar a performance (sobrevivência e crescimento) de espécies potenciais para uso em projetos de restauração, antecipando assim quais teriam melhor desempenho em campo (Roberts et al. 2010, Guzman-Luna \& Martinez-Garza 2016). Atributos de semente, por exemplo, são associados à taxa e velocidade de germinação das espécies, identificando assim quais teriam maior possibilidade de sucesso no estabelecimento inicial em áreas de restauração (Silva \&Vieira 2017). Grupos funcionais também têm sido utilizados no planejamento da restauração: categorias relacionadas a estágio sucessional (pioneiras, secundárias iniciais, secundárias tardias), modo de dispersão (zoocóricas, anemocóricas), forma de crescimento (herbáceas, arbustos, árvores), e capacidade de fixação de nitrogênio (plantas fixadoras de nitrogênio) são comumente utilizadas para o planejamento de seleção de espécies para 

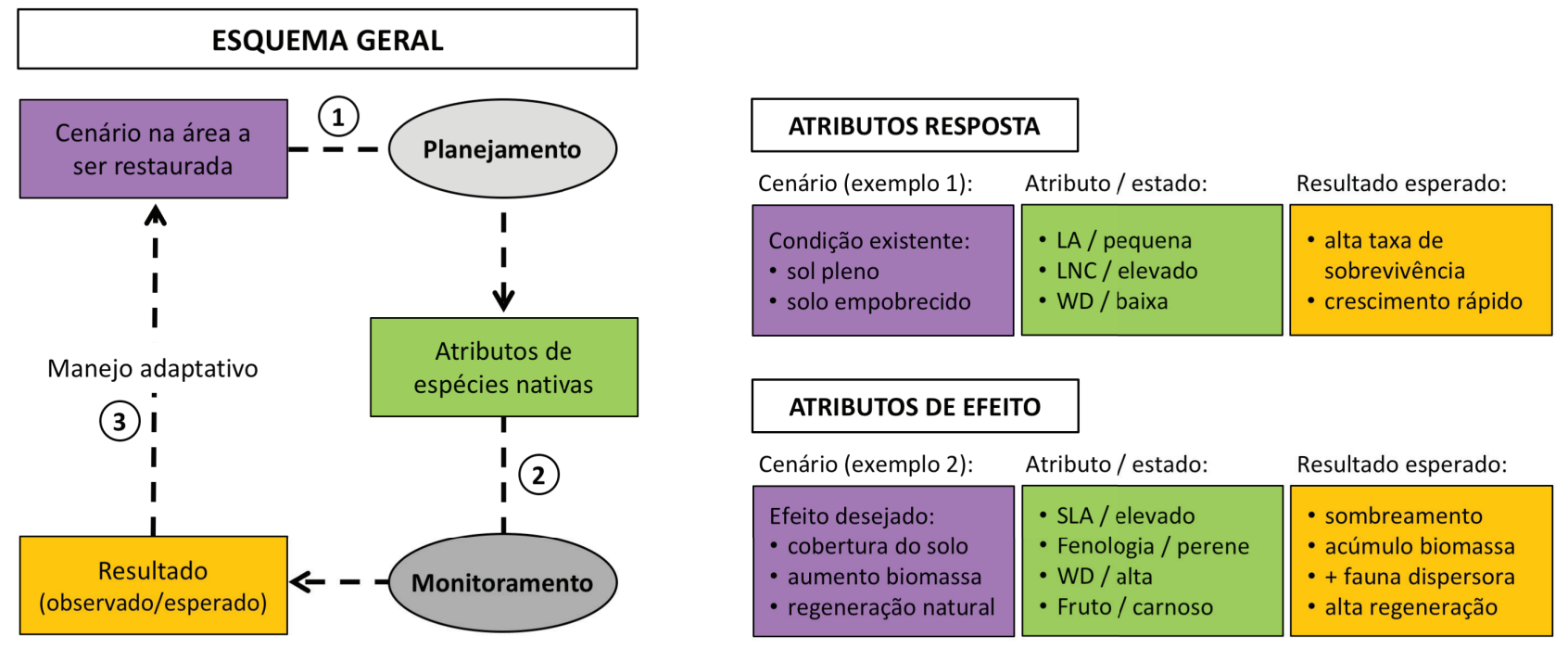

Figura 1. Diagrama esquemático ilustrando a aplicação de atributos funcionais no planejamento e monitoramento da restauração ecológica. A partir de um cenário existente na área a ser restaurada, seja ele focado na condição da área degradada ou em efeitos desejados nos processos ecossistêmicos (retângulos roxos), é possível planejar o projeto de restauração e a seleção de espécies que serão plantadas/semeadas a partir de seus atributos funcionais (1). A seleção considera atributos funcionais de interesse (atributos resposta e/ou de efeito) e seus estados/valores (retângulos verdes), considerando as condições existentes na área degradada (Cenário - exemplo 1) ou os efeitos desejados sobre processos ecossistêmicos (Cenário exemplo 2). A escolha deve considerar o conhecimento ecológico das relações entre atributos (seus estados/ valores) e variáveis associadas às condições ou aos efeitos (veja exemplos ao longo do texto). Após a seleção e estabelecimento das espécies na área em restauração, ações de monitoramento permitem avaliar os resultados esperados e observados (retângulos laranjas) e informam sobre $\mathrm{o}$ alcance das metas estabelecidas no projeto (2). Conforme a avaliação dos resultados e do cenário atual da área, é possível retornar à etapa de planejamento, assumindo a abordagem do manejo adaptativo (3) da área em restauração. Abreviações indicam: LA, área foliar; LNC, conteúdo de nitrogênio foliar; SLA, área foliar específica; WD, densidade da madeira.

Figure 1. Diagram showing the use of functional traits in the planning and monitoring stages of ecological restoration. From an existing scenario present in the area to be restored, which can refer to the condition of the degraded area or to the desired effects on ecosystem processes (purple boxes), it is possible to plan the restoration project and the selection of species to be planted/seeded based on their functional traits (1). The selection considers targeted functional traits (response or effect traits) and their attributes (green boxes), considering the existing conditions of the degraded area (Scenario - example 1) or the desired effects on ecosystem processes (Scenario-example 2). The choice should account for the ecological knowledge of the relationships between traits (their attributes) and the variables associated to conditions or effects (see text for examples). After the selection and the establishment of species in the area undergoing restoration, monitoring actions enable the evaluation of the expected and observed results (orange boxes) and inform the extent to which the aims of the restoration project are being met (2). Given the evaluation of the results and the current state of the area, it is possible to return to the planning stage, assuming the adaptive management approach (3) of the area undergoing restoration. Abbreviations refer to: $L A$, leaf area; $L N C$, leaf nitrogen content; $S L A$, specific leaf area; $W D$, wood density.

uso em restauração ecológica (de Meira et al. 2015, Melo et al. 2015).

Projetos de restauração que consideram o uso de atributos funcionais de plantas ainda na etapa de planejamento constituem estudos empíricos sobre regras de montagem de comunidades (HilleRisLambers et al. 2012) e os resultados podem ser preditos através de modelagens baseadas em atributos (Cadotte et al. 2015, Shipley et al. 2016). Esses modelos podem ser utilizados para diversos objetivos, como: predizer comunidades que potencializam uma função de interesse (foco em atributo de efeito); que aumentam a diversidade funcional da comunidade; ou que elevam o potencial competitivo das espécies nativas frente às invasoras (Laughlin 2014, Laughlin et al. 2018). $\mathrm{O}$ resultado do modelo informa ao usuário a abundância relativa de cada espécie a ser utilizada na restauração, considerando os valores/estados de seus atributos e condições/funções de interesse. $\mathrm{Ou}$ seja, os modelos geram combinações de espécies e as abundâncias de cada uma delas, 
de forma a auxiliar o restaurador a selecionar as espécies com base em seus atributos e nos alvos/ metas de restauração. A combinação de espécies para formar comunidades pode ter um efeito não apenas inicial no estabelecimento e crescimento dos indivíduos, como também pode ter um efeito que perdura no longo prazo, quando algumas espécies exercem "efeitos de prioridade" (priority effects, em inglês) e direcionam o desenvolvimento posterior da comunidade. Diversos estudos apontam que os atributos das espécies, mais que a identidade taxonômica, são relevantes para identificar combinações que podem gerar efeitos de prioridade desejados ou indesejados para o desenvolvimento da comunidade no longo prazo (Clark et al. 2012, von Gillhaussen et al. 2014, Fukami 2015, Weidlich et al. 2018). Portanto, a seleção de espécies baseada em atributos na etapa de planejamento da restauração pode influenciar diretamente o desenvolvimento dos ecossistemas restaurados.

\section{Ecologia funcional na avaliação e monitoramento da restauração}

A avaliação de projetos de restauração é feita pelo acompanhamento da área anteriormente degradada, analisando, ao longo do tempo, as mudanças de parâmetros relativos a estrutura da vegetação, riqueza/diversidade de espécies e processos ecossistêmicos (Ruiz-Jaen \& Aide 2005, Rodrigues et al. 2009). Geralmente se utilizam características do sistema de referência e/ou do sistema degradado como base para determinar o quanto a área em restauração está se modificando e em qual trajetória. A busca por indicadores do sucesso do projeto de restauração é uma atividade contínua, sujeita a intensas discussões (ver debate: Reid 2015; Suganuma \& Durigan 2015; Brancalion \& Holl 2016). Assim, é sugerida uma avaliação que considere informações referentes a estrutura da vegetação, diversidade de espécies e processos ecossistêmicos de forma conjunta (Ruiz-Jaén \& Aide 2005, Suding et al. 2015). Uma vez que o restabelecimento de funções e processos ecossistêmicos nem sempre ocorre na mesma velocidade que a recuperação da vegetação acima do solo (Cortina et al. 2006, Matzek et al. 2016) é importante que sejam medidos parâmetros relativos aos processos, de modo a avaliar a funcionalidade geral do ecossistema (ex. Rosenfield
\& Müller 2019) e sua sustentabilidade ao longo do tempo, meta comum da restauração.

Atributos funcionais podem ser utilizados como indicadores de processos/funções ecossistêmicas (Garnier et al. 2004) e diversos estudos empíricos embasam essas relações, como por exemplo: conteúdo de massa seca foliar e conteúdo de nitrogênio foliar apresentando, respectivamente, efeitos negativo e positivo na fertilidade do solo (Laughlin et al. 2015); área foliar específica afetando positivamente o incremento de biomassa (Finegan et al. 2015); e a massa de semente apresentando efeito positivo na sobrevivência de plântulas (Moles \& Westoby 2004), influenciando o potencial de regeneração dos ecossistemas. Assim, monitoramentos que contemplem a avaliação de atributos funcionais permitem analisar mudanças nos processos ecológicos e semelhanças com a funcionalidade do ecossistema de referência.

Nossa revisão sistemática revelou que atributos ou grupos funcionais são utilizados no monitoramento de diferentes formas, desde para compreender diferenças de desenvolvimento dos indivíduos (crescimento e sobrevivência) das espécies plantadas (Martínez-Garza et al. 2013, Guimaraes et al. 2018) até como indicadores do sucesso da restauração (Suganuma \& Durigan 2015). Os atributos funcionais, porém, são principalmente utilizados para avaliar as respostas da comunidade ao longo do tempo (Dzwonko \& Loster 2007, Hedberg et al. 2013, Garcia et al. 2015), que indiquem a ação de determinados filtros ambientais sobre os padrões de montagem de comunidades (Helsen et al. 2013, Hedberg et al. 2014), de distúrbios (ex. fogo) ou ainda de espécies invasoras sobre o desenvolvimento das comunidades de plantas em áreas de restauração (Laughlin et al. 2004, Fournier et al. 2015, O'Leary et al. 2018). Além disso, também são utilizados para avaliar diferenças mais específicas, comparadas aos padrões observados em áreas de referência (Suganuma \& Torezan 2013).

Nesse sentido, os atributos utilizados para monitorar trajetórias ao longo do tempo e padrões nas comunidades em restauração são diversos: foliares, de crescimento, reprodutivos, radiculares, entre outros. Atributos foliares, de caule, grupos sucessionais e de formas de crescimento em geral são utilizados para monitorar o desenvolvimento da comunidade de plantas em si (ex. Suganuma et al. 
2014, Rosenfield \& Müller 2019), enquanto atributos fenológicos e reprodutivos em geral são avaliados para inferir sobre a recuperação de outros níveis tróficos, essenciais aofuncionamento ecossistêmico (ex. Garcia et al. 2014, Montoya-Pfeiffer et al. 2018). Cabe salientar ainda que monitoramentos com base em atributos e/ou grupos funcionais, associados a padrões observados em ecossistemas de referência, permitem aprimorar técnicas de restauração e o planejamento de novos projetos, uma vez que os dados complementam o arcabouço teórico associado à restauração ecológica. Assim, resultados de monitoramento podem subsidiar novas ações de intervenção ou mesmo a redefinição de metas, calcadas no contexto local e regional das áreas sob restauração (Garcia et al. 2014, Suganuma \& Durigan 2015, Rosenfield \& Müller 2017, Toledo et al. 2018).

O monitoramento das trajetórias sucessionais também pode ser realizado através de modelagem baseada em atributos funcionais, tal como sugerido no planejamento da restauração (ver acima). Isso pode ser feito projetando a distribuição de abundâncias futura da comunidade por meio de avaliações ao longo do tempo e de comparações com a comunidade resultante do modelo, que é baseado no ecossistema de referência (Laughlin 2014). Uma vez que o modelo gera abundâncias ideais para a espécies, para se obter uma comunidade funcionalmente mais similar ao ecossistema de referência, o restaurador pode alterar a proporção de determinadas espécies. Dessa forma, o manejo pode ser realizado de forma mais direcionada com o intuito de aumentar a similaridade funcional com relação ao ecossistema de referência ou de alterar o nível de determinado processo ecológico, podendo envolver ações de adição ou remoção de espécies com características específicas. Através do monitoramento do estado dos atributos funcionais da comunidade, é possível inferir sobre trajetórias de desenvolvimento de processos ecológicos, a similaridade funcional com o ecossistema de referência e o sucesso das metas do projeto de restauração (Laughlin et al. 2017, Rosenfield \& Müller 2017).

\section{CONCLUSÃO}

O presente trabalho buscou enfatizar como a ecologia funcional pode ser utilizada para planejar e monitorar projetos de restauração ecológica no contexto da avaliação do funcionamento dos ecossistemas. Ela tem se mostrado uma ferramenta importante capaz de acelerar o desenvolvimento da vegetação, ou combater espécies invasoras, além de ter uma associação direta com diversas propriedades ecossistêmicas. Estudos básicos e experimentais que mostrem relações das características das plantas com condições ambientais e processos ecológicos podem auxiliar na montagem de comunidades e na avaliação do funcionamento de áreas em restauração e da sua similaridade com áreas de referência. Frente ao cenário atual de mudanças globais climáticas e de uso do solo, é importante focar não só na composição florística das comunidades, mas principalmente nas características funcionais do ecossistema, assegurando seu desenvolvimento em longo prazo, possibilitando interações entre as espécies e a provisão de serviços ecossistêmicos. Compreender essas relações ecológicas permite uma maior efetividade na aplicação de recursos, aumentando o sucesso das ações de restauração ecológica. Nesse sentido, uma maior aproximação entre a academia e praticantes da restauração também se faz necessária, de modo a interligar conhecimentos teóricos com a prática da restauração. A disseminação de materiais didáticos para a restauração ecológica, baseada em atributos funcionais das plantas, tem a possibilidade de abranger diferentes ecossistemas e problemáticas, independente de regiões específicas.

\section{AGRADECIMENTOS}

Este estudo se consolidou ao longo do doutorado de MFR, tendo apoio financeiro da Coordenação de Aperfeiçoamento de Pessoal de Nível Superior, Brasil (CAPES; Código de Financiamento 001). Também agradecemos ao Conselho Nacional de Desenvolvimento Científico e Tecnológico (CNPq) pela bolsa de pesquisa à SCM (Processo 309874/2015-7).

\section{REFERÊNCIAS}

Bird, R. B., \& Nimmo, D. 2018. Restore the lost ecological functions of people. Nature Ecology \& Evolution, 2, 1050-1052. DOI: https://doi. org/10.1038/s41559-018-0576-5

Bochet, E., \& Garcia-Fayos, P. 2015. Identifying 
plant traits: A key aspect for species selection in restoration of eroded roadsides in semiarid environments. Ecological Engineering, 83, 444451. DOI: 10.1016/j.ecoleng.2015.06.019

Brancalion, P. H. S., \& Holl, K. D. 2016. Functional composition trajectory: a resolution to the debate between Suganuma, Durigan, and Reid. Restoration Ecology, 24(1), 1-3. DOI: 10.1111/ rec. 12312

Buchmann, T., Schumacher, J., Ebeling, A., Eisenhauer, N., Fischer, M., Gleixner, G., Hacker, N., Lange, M., Oelmann, Y., Schulze, E.-D., Weigelt, A., Weisser, W. W., Wilcke, W., \& Roscher, C. 2018. Connecting experimental biodiversity research to real-world grasslands. Perspectives in Plant Ecology, Evolution and Systematics, 33, 78-88. DOI: doi.org/10.1016/j. ppees.2018.06.002

Cadotte, M. W., Arnillas, C. A., Livingstone, S. W., \& Yasui, S. L. E. 2015. Predicting communities from functional traits. Trends in Ecology and Evolution, 30(9), 510-511. DOI: 10.1016/j. tree.2015.07.001

Calmon, M., Brancalion, P. H. S., Paese, A., Aronson, J., Castro, P., Silva, S. C., \& Rodrigues, R. R. 2011. Emerging Threats and Opportunities for LargeScale Ecological Restoration in the Atlantic Forest of Brazil. Restoration Ecology, 19(2), 154158. DOI: 10.1111/j.1526-100X.2011.00772.x

Cardinale, B. J., Wright, J. P., Cadotte, M.W., Carroll, I. T., Hector, A., Srivastava, D. S., Loreau, M., \&Weis, J. J. 2007. Impacts of plant diversity on biomass production increase through time because of species complementarity. Proceedings of the National Academy of Sciences, 104(46), 1812318128. DOI: 10.1073/pnas.0709069104

Chave, J., Coomes, D., Jansen, S., Lewis, S. L., Swenson, N. G., \& Zanne, A. E. 2009. Towards a worldwide wood economics spectrum. Ecology Letters, 12(4), 351-366. DOI: 10.1111/j.14610248.2009.01285.x

Chaves, R. B., Durigan, G., Brancalion, P. H. S., \& Aronson, J. 2015. On the need of legal frameworks for assessing restoration projects success: New perspectives from São Paulo state (Brazil). Restoration Ecology, 23(6), 754-759. DOI: $10.1111 /$ rec. 12267

Chazdon, R. L., Brancalion, P. H. S., Lamb, D., Laestadius, L., Calmon, M., \& Kumar, C. 2017. A policy-driven knowledge agenda for global forest and landscape restoration. Conservation Letters, 10(1), 125-132. DOI: 10.1111/conl.12220 Clark, D. L., Wilson, M., Roberts, R., Dunwiddie, P. W., Stanley, A., \& Kaye, T. N. 2012. Plant traits - a tool for restoration? Applied Vegetation Science, 15, 449-458. DOI: 10.1111/j.1654109X.2012.01198.X

Cortina, J., Maestre, F. T., Vallejo, R., Baeza, M. J., Valdecantos, A., \& Pérez-Devesa, M. 2006. Ecosystem structure, function, and restoration success: Are they related? Journal for Nature Conservation, 14(3-4), 152-160. DOI: 10.1016/j. jnc.2006.04.004

de Bello, F., Lavorel, S., Díaz, S., Harrington, R., Cornelissen, J. H. C., Bardgett, R. D., Berg, M. P., Cipriotti, P., Feld, C. K., Hering, D., da Silva, P. M., Potts, S. G., Sandin, L., Sousa, J. P., Storkey, J., Wardle, D. A., \& Harrison, P. A. 2010. Towards an assessment of multiple ecosystem processes and services via functional traits. Biodiversity and Conservation, 19(10), 2873-2893. DOI: 10.1007/s10531-010-9850-9

de Meira, M., Pereira, I., Machado, E., Mota, S., \& Otoni, T. 2015. Potential species for recovery areas semideciduous forest in iron exploration in the Serra Espinhaco. Bioscience Journal, 31(1), 283-295.

Díaz, S., Lavorel, S., de Bello, F., Quetier, F., Grigulis, K., \& Robson, T. M. 2007. Incorporating plant functional diversity effects in ecosystem service assessments. Proceedings of the National Academy of Sciences, 104(52), 20684-20689. DOI: $10.1073 /$ pnas.0704716104

Durigan, G., \& Ramos, V. S. 2013. Manejo Adaptativo: primeiras experiências na Restauração de Ecossistemas. São Paulo, Brazil: p. 50.

Dzwonko, Z., \& Loster, S. 2007. A functional analysis of vegetation dynamics in abandoned and restored limestone grasslands. Journal of Vegetation Science, 18(2), 203-212. DOI: 10.1111/j.1654-1103.2007.tb02531.x

Finegan, B., Peña-Claros, M., de Oliveira, A., Ascarrunz, N., Bret-Harte, M. S., CarreñoRocabado, G., Casanoves, F., Díaz, S., Eguiguren Velepucha, P., Fernandez, F., Licona, J. C., Lorenzo, L., Salgado Negret, B., Vaz, M., \& Poorter, L. 2015. Does functional trait diversity predict above-ground biomass and productivity of tropical forests? Testing three alternative hypotheses. Journal of Ecology, 103(1), 191-201. 
DOI: $10.1111 / 1365-2745.12346$

Fournier, B., Gillet, F., Le Bayon, R., Mitchell, E., \& Moretti, M. 2015. Functional responses of multitaxa communities to disturbance and stress gradients in a restored floodplain. Journal of Applied Ecology, 52(5), 1364-1373. DOI: 10.1111/1365-2664.12493

Freschet, G. T., Aerts, R., \& Cornelissen, J. H. C. 2012. A plant economics spectrum of litter decomposability. Functional Ecology, 26(1), 5665. DOI: 10.1111/j.1365-2435.2011.01913.x

Fujii, S., Mori, A. S., Koide, D., Makoto, K., Matsuoka, S., Osono, T., \& Isbell, F. 2017. Disentangling relationships between plant diversity and decomposition processes under forest restoration. Journal of Applied Ecology, 54, 80-90. DOI: 10.1111/1365-2664.12733

Fukami, T. 2015. Historical Contingency in Community Assembly: Integrating Niches, Species Pools, and Priority Effects. Annual Review of Ecology, Evolution, and Systematics, 46, 1-23. DOI: 10.1146/annurev-ecolsys-110411-160340

Galetti, M., Pizo, M. A., \& Morellato, L. P. C. 2011. Diversity of functional traits of fleshy fruits in a species-rich Atlantic rain forest. Biota Neotropica, 11(1), 181-194. DOI: 10.1590/ S1676-06032011000100019

Garcia, Letícia C., Hobbs, R. J., Santos, F. A. M., \& Rodrigues, R. R. 2014. Flower and Fruit Availability along a Forest Restoration Gradient. Biotropica, 46(1), 114-123. DOI: 10.1111/ btp. 12080

Garcia, Letícia Couto, Cianciaruso, M. V., Ribeiro, D. B., dos Santos, F. A. M., \& Rodrigues, R. R. 2015. Flower functional trait responses to restoration time. Applied Vegetation Science, 18(3), 402412. DOI: 10.1111 /avsc. 12163

Garnier, E., Cortez, J., Billès, G., Navas, M. L., Roumet, C., Debussche, M., Laurent, G., Blanchard, A., Aubry, D., Bellmann, A., Neill, C., \& Toussaint, J. P. 2004. Plant functional markers capture ecosystem properties during secondary succession. Ecology, 85(9), 2630-2637. DOI: 10.1890/03-0799

Garnier, E., Navas, M.-L., \& Grigulis, K. 2016. Plant Functional Diversity - organisms traits, community structure, and ecosystem properties. Oxford, UK: Oxford University Press: p. 231.

Grime, J. P. 1998. Benefits of plant diversity to ecosystems: immediate, filter and founder effects. Journal of Ecology, 86, 902-910. DOI: 10.1046/j.1365-2745.1998.00306.x

Guimaraes, Z., dos Santos, V., Nogueira, W., Martins, N., \& Ferreira, M. 2018. Leaf traits explaining the growth of tree species planted in a Central Amazonian disturbed area. Forest Ecology and Management, 430, 618-628. DOI: 10.1016/j. foreco.2018.08.048

Guzman-Luna, A., \& Martinez-Garza, C. 2016. Performance of 15 tropical tree species recruited or transplanted on restoration settings. Botanical Sciences, 94(4), 757-773. DOI: $10.17129 /$ botsci.659

Hallett, L., Chapple, D., Bickart, N., Cherbowsky, A., Fernandez, L., Ho, C., Alexander, M., Schwab, K., \& Suding, K. 2017. Trait Complementarity Enhances Native Plant Restoration in an Invaded Urban Landscape. Ecological Restoration, 35(2), 148-155. DOI: 10.3368/er.35.2.148

Hedberg, P., Kozub, L., \& Kotowski, W. 2014. Functional diversity analysis helps to identify filters affecting community assembly after fen restoration by top-soil removal and hay transfer. Journal of Nature Conservation, 22(1), 50-58. DOI: 10.1016/j.jnc.2013.08.004

Hedberg, P., Saetre, P., Sundberg, S., Rydin, H., \& Kotowski, W. 2013. A functional trait approach to fen restoration analysis. Applied Vegetation Science, 16(4), 658-666. DOI: 10.1111/ avsc. 12042

Helsen, K., Hermy, M., \& Honnay, O. 2013. Spatial isolation slows down directional plant functional group assembly in restored seminatural grasslands. Journal of Applied Ecology, 50, 404-413. DOI: 10.1111/1365-2664.12037

Hiers, J. K., Mitchell, R. J., Barnett, A., Walters, J. R., MacK, M., Williams, B., \& Sutter, R. 2012. The dynamic reference concept: Measuring restoration success in a rapidly changing noanalogue future. Ecological Restoration, 30(1), 27-36. DOI: 10.3368/er.30.1.27

Higgs, E., Falk, D. A., Guerrini, A., Hall, M., Harris, J., Hobbs, R. J., Jackson, S. T., Rhemtulla, J. M., \& Throop, W. 2014. The changing role of history in restoration ecology. Frontiers in Ecology and the Environment, 12(9), 499-506. DOI: $10.1890 / 110267$

HilleRisLambers, J., Adler, P. B., Harpole, W. S., Levine, J. M., \& Mayfield, M. M. 2012. Rethinking Community Assembly through the Lens of 
Coexistence Theory. Annual Review of Ecology, Evolution, and Systematics, 43, 227-248. DOI: 10.1146/annurev-ecolsys-110411-160411

Holl, K. D., \& Aide, T. M. 2011. When and where to actively restore ecosystems? Forest Ecology and Management, 261(10), 1558-1563. DOI: 10.1016/j.foreco.2010.07.004

Holl, Karen D., Zahawi, R. a., Cole, R. J., Ostertag, R., \& Cordell, S. 2011. Planting seedlings in tree islands versus plantations as a large-scale tropical forest restoration strategy. Restoration Ecology, 19(4), 470-479. DOI: 10.1111/j.1526100X.2010.00674.X

Hooper, D. U., Chapin, III, F. S., Ewel, J. J., Hector, A., Inchausti, P., Lavorel, S., Lawton, J. H., Lodge, D. M., Loreau, M., Naeem, S., Schmid, B., Setala, H., Symstad, A. J., Vandermeer, J., \& Wardle, D. A. 2005. Effects of biodiversity on ecosystem functioning: a consensus of current knowledge. Ecological Monographs, 75(1), 3-35. DOI: 10.1890/04-0922

Jochum, M., Fischer, M., Isbell, F., Roscher, C., van der Plas, F., Boch, S., Boenisch, G., Buchmann, N., Catford, J. A., Cavender-Bares, J., Ebeling, A., Eisenhauer, N., Gleixner, G., Hölzel, N., Kattge, J., Klaus, V. H., Kleinebecker, T., Lange, M., Le Provost, G., Meyer, S. T., Molina-Venegas, R., Mommer, L., Oelmann, Y., Penone, C., Prati, D., Reich, P. B., Rindisbacher, A., Schäfer, D., Scheu, S., Schmid, B., Tilman, D., Tscharntke, T., Vogel, A., Wagg, C., Weigelt, A., Weisser, W.W., Wilcke, W., \& Manning, P. 2019. The results of biodiversityecosystem functioning experiments are realistic. BioRxiv, 725812. DOI: 10.1101/725812

Kageyama, P. Y., Oliveira, R. E., Moraes, L. F. D., Engel, V. L., \& Gandara, F. B. 2003. Restauração ecológica de ecossistemas naturais. P. Y. Kageyama R. E. Oliveira L. F. D. Moraes V. L. Engel \& F. B. Gandara (Eds.), Botucatu - SP: Fundação de Estudos e Pesquisas Agrícolas e Florestais FEPAF: p. 340.

Kattge, J., Díaz, S., Lavorel, S., Prentice, I. C., Leadley, P., Bönisch, G., Garnier, E., Westoby, M., Reich, P. B., Wright, I. J., Cornelissen, J. H. C., Violle, C., Harrison, S. P., Van Bodegom, P. M., Reichstein, M., Enquist, B. J., Soudzilovskaia, N. A., Ackerly, D. D., Anand, M., Atkin, O., Bahn, M., Baker, T. R., Baldocchi, D., Bekker, R., Blanco, C. C., Blonder, B., Bond, W. J., Bradstock, R., Bunker, D. E., Casanoves, F., Cavender-Bares, J.,
Chambers, J. Q., Chapin, F. S., Chave, J., Coomes, D., Cornwell, W. K., Craine, J. M., Dobrin, B. H., Duarte, L., Durka, W., Elser, J., Esser, G., Estiarte, M., Fagan, W. F, Fang, J., Fernández-Méndez, F, Fidelis, A., Finegan, B., Flores, O., Ford, H., Frank, D., Freschet, G. T., Fyllas, N. M., Gallagher, R. V., Green, W. A., Gutierrez, A. G., Hickler, T., Higgins, S. I., Hodgson, J. G., Jalili, A., Jansen, S., Joly, C. A., Kerkhoff, A. J., Kirkup, D., Kitajima, K., Kleyer, M., Klotz, S., Knops, J. M. H., Kramer, K., Kühn, I., Kurokawa, H., Laughlin, D., Lee, T. D., Leishman, M., Lens, F, Lenz, T., Lewis, S. L., Lloyd, J., Llusià, J., Louault, F., Ma, S., Mahecha, M. D., Manning, P., Massad, T., Medlyn, B. E., Messier, J., Moles, A. T., Müller, S. C., Nadrowski, K., Naeem, S., Niinemets, Ü., Nöllert, S., Nüske, A., Ogaya, R., Oleksyn, J., Onipchenko, V. G., Onoda, Y., Ordoñez, J., Overbeck, G., Ozinga, W. A., Patiño, S., Paula, S., Pausas, J. G., Peñuelas, J., Phillips, O. L., Pillar, V., Poorter, H., Poorter, L., Poschlod, P., Prinzing, A., Proulx, R., Rammig, A., Reinsch, S., Reu, B., Sack, L., Salgado-Negret, B., Sardans, J., Shiodera, S., Shipley, B., Siefert, A., Sosinski, E., Soussana, J. F., Swaine, E., Swenson, N., Thompson, K., Thornton, P., Waldram, M., Weiher, E., White, M., White, S., Wright, S. J., Yguel, B., Zaehle, S., Zanne, A. E., \& Wirth, C. 2011. TRY - a global database of plant traits. Global Change Biology, 17(9), 2905-2935. DOI: 10.1111/j.1365-2486.2011.02451.x

Kollmann, J., Meyer, S. T., Bateman, R., Conradi, T., Gossner, M. M., Mendonça Jr., M. de S., Fernandes, G. W., Hermann, J. M., Koch, C., Müller, S. C., Oki, Y., Overbeck, G. E., Paterno, G. B., Rosenfield, M. F., Toma, T. S. P., \& Weisser, W. W. 2016. Integrating ecosystem functions into restoration ecology - recent advances and future directions. Restoration Ecology, 24(6), 722-730. DOI: $10.1111 /$ rec.12422

Laughlin, D., Bakker, J., Stoddard, M., Daniels, M., Springer, J., Gildar, C., Green, A., \& Covington, W. 2004. Toward reference conditions: wildfire effects on flora in an old-growth ponderosa pine forest. Forest Ecology and Management, 199(1), 137-152. DOI: 10.1016/j.foreco.2004.05.034

Laughlin, D. C. 2014. Applying trait-based models to achieve functional targets for theory-driven ecological restoration. Ecology Letters, 17(7), 771-784. DOI: 10.1111 /ele.12288

Laughlin, D. C., Chalmandrier, L., Joshi, C., 
Renton, M., Dwyer, J. M., \& Funk, J. L. 2018. Generating species assemblages for restoration and experimentation: A new method that can simultaneously converge on average trait values and maximize functional diversity. Methods in Ecology and Evolution, 9, 1764-1771. DOI: 10.1111/2041-210X.13023

Laughlin, D. C., Leppert, J. J., Moore, M. M., \& Sieg, C. H. 2010. A multi-trait test of the leaf-height-seed plant strategy scheme with 133 species from a pine forest flora. Functional Ecology, 24(3), 493501. DOI: 10.1111/j.1365-2435.2009.01672.x

Laughlin, D. C., Richardson, S. J., Wright, E. F., \& Bellingham, P. J. 2015. Environmental Filtering and Positive Plant Litter Feedback Simultaneously Explain Correlations Between Leaf Traits and Soil Fertility. Ecosystems, 18(7), 1269-1280. DOI: 10.1007/s10021-015-9899-0

Laughlin, D., Strahan, R., Moore, M., Fule, P., Huffman, D., \& Covington, W. 2017. The hierarchy of predictability in ecological restoration: are vegetation structure and functional diversity more predictable than community composition? Journal of Applied Ecology, 54(4), 1058-1069. DOI: $10.1111 / 1365-2664.12935$

Lavorel, S., \& Garnier, E. 2002. Predicting changes in community composition and ecosystem functioning from plant traits: revisiting the Holy Grail. Functional Ecology, 16(5), 545-556. DOI: 10.1046/j.1365-2435.2002.00664.x

Lavorel, Sandra. 2013. Plant functional effects on ecosystem services. Journal of Ecology, 101(1), 4-8. DOI: 10.1111/1365-2745.12031

Loreau, M, Naeem, S., Inchausti, P., Bengtsson, J., Grime, J. P., Hector, A., Hooper, D. U., Huston, M. A., Raffaelli, D., Schmid, B., Tilman, D., \& Wardle, D. A. 2001. Biodiversity and Ecosystem Functioning: Current Knowledge and Future Challenges. Science, 294, 804-809. DOI: 10.1126/ science. 1064088

Loreau, Michel. 1998. Biodiversity and ecosystem functioning: A mechanistic model. Proceedings of the National Academy of Sciences, 95, 56325636. DOI: $10.1073 /$ pnas.95.10.5632

Loreau, Michel, \& Hector, A. 2001. Partitioning selection and complementarity in biodiversity experiments. Nature, 412, 72-76. DOI: $10.1038 / 35083573$

Walter V. Reid, Harold A. Mooney, Angela Cropper, Doris Capistrano, Stephen R. Carpenter,
Kanchan Chopra, Partha Dasgupta, Thomas Dietz, Anantha Kumar Duraiappah, Rashid Hassan, Roger Kasperson, Rik Leemans, Robert M. May, Tony (A.J.) McMichael, Prabhu Pingali, Cristián Samper, Robert Scholes, Robert T. Watson, A.H. Zakri, Zhao Shidong, Neville J. Ash, Elena Bennett, Pushpam Kumar, Marcus J. Lee, Ciara Raudsepp-Hearne, Henk Simons, Jillian Thonell, and Monika B. Zurek. 2005. Ecosystems and Human Well-being: Biodiversity Synthesis. MA (Millennium Ecosystem Assessment). Island Press, Washington, DC

Martínez-Garza, C., Bongers, F., \& Poorter, L. 2013. Are functional traits good predictors of species performance in restoration plantings in tropical abandoned pastures? Forest Ecology and Management, 303, 35-45. DOI: 10.1016/j. foreco.2013.03.046

Mason, N. W. H., Mouillot, D., Lee, W. G., \&Wilson, J. B. 2005. Functional richness, functional and functional evenness divergence: the primary of functional components diversity. Oikos, 111(1), 112-118. DOI: 10.1111/j.0030-1299.2005.13886.x

Matzek, V., Warren, S., \& Fisher, C. 2016. Incomplete recovery of ecosystem processes after two decades of riparian forest restoration. Restoration Ecology, 24(5), 637-645. DOI: $10.1111 /$ rec.12361

McDonald, T., Gann, G. D., Jonson, J., \& Dixon, K.W. 2016. International standards for the practice of ecological restoration - including principles and key concepts. Society for Ecological Restoration, Washington, D.C.

Melo, A., Daronco, C., Re, D., \& Durigan, G. 2015. Tree species attributes and facilitation of natural regeneration in heterogeneous planting of riparian vegetation. Scientia Forestalis, 43(106), 333-344.

Mesquita, R. D. C. G., Massoca, P. E. D. S., Jakovac, C. C., Bentos, T. V., \& Williamson, G. B. 2015. Amazon rain forest succession: stochasticity or land-use legacy? BioScience, 65(9), 849-861. DOI: $10.1093 /$ biosci/biv108

Moles, A. T., \& Westoby, M. 2004. Seedling survival and seed size: a synthesis of the literature. Journal of Ecology, 92(3), 372-383. DOI: 10.1111/j.00220477.2004.00884.x

Montoya-Pfeiffer, P., Rodrigues, R., Metzger, J., da Silva, C., Baquero, O., \& dos Santos, I. 2018. Are the assemblages of tree pollination modes 
being recovered by tropical forest restoration? Applied Vegetation Science, 21(1), 156-163. DOI: 10.1111/avsc. 12335

Norden, N., Angarita, H. A., Bongers, F., MartínezRamos, M., Granzow-de la Cerda, I., van Breugel, M., Lebrija-Trejos, E., Meave, J. A., Vandermeer, J., Williamson, G. B., Finegan, B., Mesquita, R., \& Chazdon, R. L. 2015. Successional dynamics in Neotropical forests are as uncertain as they are predictable. Proceedings of the National Academy of Sciences of the United States of America, 112(26), 8013-8018. DOI: 10.1073/ pnas. 1500403112

O’Leary, B., Burd, M., Venn, S., \& Gleadow, R. 2018. Integrating the Passenger-Driver hypothesis and plant community functional traits to the restoration of lands degraded by invasive trees. Forest Ecology and Management, 408, 112-120. DOI: 10.1016/j.foreco.2017.10.043

Oliveira, L. Z., Uller, H. F., Klitzke, A. R., Eleotério, J. R., \&Vibrans, A. C. 2019. Towards the Fulfillment of a Knowledge Gap:Wood Densities for Species of the Subtropical Atlantic Forest. Data, 4(3), 104. DOI: $10.3390 /$ data 4030104

Ostertag, R., Warman, L., Cordell, S., \& Vitousek, P. M. 2015. Using plant functional traits to restore Hawaiian rainforest. Journal of Applied Ecology, 52, 805-809. DOI: 10.1111/1365-2664.12413

Overbeck, G. E., Rosenfield, M. F., Vieira, M. S., \& Müller, S. C. 2016. Princípios e desafios da restauração ecológica em ecossistemas brasileiros. In: A. L. Peixoto, J. R. P. Luz, \& M. A. de Brito (Eds.), Conhecendo a biodiversidade. pp. 141-155. Brasília: MCTIC, CNPq, PPBio.

Perez-Harguindeguy, N., Díaz, S., Garnier, E., Lavorel, S., Poorter, H., Jaureguiberry, P., BretHarte, M. S., Cornwell, W. K., Craine, J. M., Gurvich, D. E., Urcelay, C., Veneklaas, E. J., Reich, P. B., Poorter, L., Wright, I. J., Ray, P., Enrico, L., Pausas, J. G., deVos, A. C., Buchmann, N., Funes, G., Quétier, F., Hodgson, J. G., Thompson, K., Morgan, H. D., ter Steege, H., van der Heijden, M. G. A., Sack, L., Blonder, B., Poschlod, P., Vaieretti, M. V., Conti, G., Staver, A. C., Aquino, S., \& Cornelissen, J. H. C. 2013. New handbook for measurement of plant functional traits worldwide. Australian Journal of Botany, 61, 167-234. DOI: 10.1071/BT12225

Reid, J. L. 2015. Indicators of success should be sensitive to compositional failures: reply to
Suganuma and Durigan. Restoration Ecology, 23(5), 519-520. DOI: 10.1111/rec.12254

Ricotta, C., \& Moretti, M. 2011. CWM and Rao's quadratic diversity: a unified framework for functional ecology. Oecologia, 167(1), 181-188. DOI: 10.1007/s00442-011-1965-5

Roberts, R., Clark, D., \& Wilson, M. 2010. Traits, neighbors, and species performance in prairie restoration. Applied Vegetation Science, 13(3), 270-279.DOI: 10.1111/j.1654-109X.2009.01073.x

Rodrigues, R R, \& Leitão Filho, H. F. 2001. Matas ciliares: conservação e recuperação. $\mathrm{R} \quad \mathrm{R}$ Rodrigues \& H. F. Leitão Filho (Eds.), São Paulo, Brazil: Editora da Universidade de São Paulo Fapesp: p. 320.

Rodrigues, Ricardo R., Lima, R. A. F., Gandolfi, S., \& Nave, A. G. 2009. On the restoration of high diversity forests: 30 years of experience in the Brazilian Atlantic Forest. Biological Conservation, 142(6), 1242-1251. DOI: 10.1016/j. biocon.2008.12.008

Rosenfield, M. F., \& Müller, S. C. 2017. Predicting restored communities based on reference ecosystems using a trait-based approach. Forest Ecology and Management, 391, 176-183. DOI: 10.1016/j.foreco.2017.02.024

Rosenfield, M. F., \& Müller, S. C. 2019. Assessing ecosystem functioning in forests undergoing restoration. Restoration Ecology, 27(1), 158-167. DOI: $10.1111 /$ rec. 12828

Rosenfield, M. F., \& Müller, S. C. 2020. Plant Traits Rather than Species Richness Explain Ecological Processes in Subtropical Forests. Ecosystems, 23, 52-66. DOI: 10.1007/s10021-019-00386-6

Ruiz-Jaen, M. C., \& Aide, T. M. 2005. Restoration success: how is it being measured? Restoration Ecology, 13(3), 569-577. DOI: 10.1111/j.1526100X.2005.00072.X

Ruiz-Jaén, M. C., \& Aide, T. M. 2005. Vegetation structure, species diversity, and ecosystem processes as measures of restoration success. Forest Ecology and Management, 218(1-3), 159173. DOI: $10.1016 /$ j.foreco.2005.07.008

Ruiz-Jaen, M. C., \& Potvin, C. 2010. Tree diversity explains variation in ecosystem function in a neotropical forest in Panama. Biotropica, 42(6), 638-646. DOI: 10.1111/j.1744-7429.2010.00631.x SER (Society for Ecological Restoration International Science \& Policy Working Group). 2004. The SER international primer on ecological restoration. 
Society for Ecological Restoration International Science \& Policy Working Group. p. 13. Tucson, Arizona.

Shipley, B., de Bello, F., Cornelissen, J. H. C., Laliberté, É., Laughlin, D. C., \& Reich, P. B. 2016. Reinforcing foundation stones in trait-based plant ecology. Oecologia, 180(4), 923-931. DOI: 10.1007/s00442-016-3549-x

Silva, R., \& Vieira, D. 2017. Direct seeding of 16 Brazilian savanna trees: responses to seed burial, mulching and an invasive grass. Applied Vegetation Science, 20(3), 410-421. DOI: $10.1111 /$ avsc. 12305

Suding, K. N., Higgs, E., Palmer, M., Callicott, J. B., Anderson, C. B., Baker, M., Gutrich, J. J., Hondula, K. L., LaFevor, M. C., Larson, B. M. H., Randall, A., Ruhl, J. B., \& Schwartz, K. Z. S. 2015. Committing to ecological restoration. Science, 348(6235), 638-640. DOI: 10.1126/science. aaa4216

Suganuma, M S, Assis, G. B. De, \& Durigan, G. 2014. Changes in plant species composition and functional traits along the successional trajectory of a restored patch of Atlantic Forest. Community Ecology, 15(1), 27-36. DOI: 10.1556/ ComEc.15.2014.1.3

Suganuma, Marcio S., \& Durigan, G. 2015. Indicators of restoration success in riparian tropical forests using multiple reference ecosystems. Restoration Ecology, 23(3), 238-251. DOI: 10.1111/rec. 12168

Suganuma, Marcio Seiji, \& Torezan, J. M. D. 2013. Evolução dos processos ecossistêmicos em reflorestamentos da Floresta Estacional Semidecídua. Hoenea, 40(3), 557-565.

TEEB. 2010. The Economics of Ecosystems and Biodiversity Ecological and Economic Foundations. Pushpam Kumar (Ed.). Earthscan, London and Washington. p. 422.

Tilman, D., Isbell, F., \& Cowles, J. M. 2014. Biodiversity and Ecosystem Functioning. Annual Review of Ecology and Systematics, 45, 471-493. DOI: 10.1126/science.1064088

Tilman, D., Knops, J., Wedin, D., Reich, P., Ritchie, M., \& Siemann, E. 1997. The influence of functional diversity and composition on ecosystem processes. Science, 277, 1300-1302. DOI: 10.1126 /science.277.5330.1300

Tilman, D., Wedin, D., \& Knops, J. 1996. Productivity and sustainability influenced by biodiversity in grassland ecosystems. Nature, 379(6567), 718720. DOI: $10.1038 / 379718 \mathrm{a} 0$

Toledo, R., Santos, R., Baeten, L., Perring, M., \& Verheyen, K. 2018. Soil properties and neighbouring forest cover affect above-ground biomass and functional composition during tropical forest restoration. Applied Vegetation Science, 21(2), 179-189. DOI: 10.1111/ avsc. 12363

Violle, C., Navas, M. L., Vile, D., Kazakou, E., Fortunel, C., Hummel, I., \& Garnier, E. 2007. Let the concept of trait be functional! Oikos, 116(5), 882-892. DOI: 10.1111/j.2007.0030-1299.15559.x

von Gillhaussen, P., Rascher, U., Jablonowski, N. D., Plückers, C., Beierkuhnlein, C., \& Temperton, V. M. 2014. Priority Effects of Time of Arrival of Plant Functional Groups Override Sowing Interval or Density Effects: A Grassland Experiment. PLoS ONE, 9(1), e86906. DOI: 10.1371/journal. pone.0086906

Weidlich, E. W. A., von Gillhaussen, P., Max, J. F. J., Delory, B. M., Jablonowski, N. D., Rascher, U., \& Temperton, V. M. 2018. Priority effects caused by plant order of arrival affect below-ground productivity. Journal of Ecology, 106, 774-780. DOI: $10.1111 / 1365-2745.12829$

Wright, I. J., Dong, N., Maire, V., Prentice, I. C., Westoby, M., Díaz, S., Gallagher, R. V., Jacobs, B. F., Kooyman, R., Law, E. A., Leishman, M. R., Niinemets, Ü., Reich, P. B., Sack, L., Villar, R., Wang, H., \& Wilf, P. 2017. Global climatic drivers of leaf size. Science, 357, 917-921. DOI: 10.1126/ science.aal 4760

Young, T. P. 2000. Restoration ecology and conservation biology. Biological Conservation, 92(1), 73-83. DOI: 10.1016/s00063207(99)00057-9

Zhu, H., Fu, B., Wang, S., Zhu, L., Zhang, L., Jiao, L., \& Wang, C. 2015. Reducing soil erosion by improving community functional diversity in semi-arid grasslands. Journal of Applied Ecology, 52(4), 1063-1072. DOI: 10.1111/13652664.12442 
Material Suplementar. Tabela A1: Síntese do número de estudos conforme cada categoria abordada nessa revisão

Tabela A2: Lista de artigos incluídos na revisão sistemática (129 artigos).

Supplementary Material. Table A1: Synthesis of the number of studies according to each category addressed in this review.

Table A2: List of articles included in the systematic review (129 articles).

Submitted: 06 May 2019 Accepted: 29 January 2020 Published on line: 18 March 2020 Associate Editor: Alan Braz 